\title{
Comparison of Bacteroides thetaiotaomicron and Escherichia coli 16S rRNA gene expression signals
}

\begin{abstract}
Correspondence
Ann M. Stevens

ams@vt.edu
\end{abstract}

Received 21 January 2009

Revised 30 April 2009

Accepted 12 May 2009

\author{
Matthew D. Mastropaolo,t‡ Mary L. Thorsont and Ann M. Stevens \\ Department of Biological Sciences, Virginia Tech, 219 Life Sciences 1, Washington Street, \\ Blacksburg, VA 24061-0910, USA
}

\begin{abstract}
There are barriers to cross-expression of genes between Bacteroides spp. and Escherichia coli. In this study, a lux-based reporter system was developed for Bacteroides and used to compare the promoter structure and function of a Bacteroides thetaiotaomicron 4001 (BT4001) 16S rRNA promoter with those of $E$. coli in vivo. Analysis of the BT4001 sequences upstream of the $16 \mathrm{~S}$ rRNA gene revealed the same overall structure known for $E$. coli $16 \mathrm{~S}$ rRNA promoters in that there were two promoters separated by $\sim 150 \mathrm{bp}$. However, the BT4001 16S rRNA promoter contains the proposed Bacteroides -7 and -33 consensus sequences instead of the $E$. coli -10 and -35 consensus sequences. The biological activity of various configurations of the BT4001 $16 \mathrm{~S}$ rRNA promoter was analysed. Experiments pairing the BT4001 16S rRNA promoter with an E. coli RBS, and vice-versa, confirmed that gene expression between the two species is restricted at the level of transcription. In Bacteroides, a difference in translation initiation also appears to limit expression of foreign genes.
\end{abstract}

\section{INTRODUCTION}

Bacteroides spp. make up a large portion of the microbial flora in the human colon, and some species are the primary cause of anaerobic infections in humans (Werner, 1974). They are as phylogenetically distinct from Gram-negative $\gamma$-proteobacterial enteric bacteria as they are from Grampositive bacteria. There are barriers that impede successful expression of genes from Escherichia coli and other Gramnegative species in Bacteroides (Smith et al., 1992). It is thought that this difference in gene expression lies in part at the level of transcription initiation and is due to the sequences within the promoter region itself. In Bacteroides, two conserved regions analogous to the -10 and -35 housekeeping consensus sequences in $E$. coli have been found, but their sequences are significantly different (Fig. 1a). One region, TTTG, is centred at -33 , and the other, TAnnTTTG, is centred at -7 (Bayley et al., 2000). Interestingly, a promoter consensus sequence similar to that of Bacteroides has also been found in Flavobacterium hibernum, another member of the phylum Bacteroidetes, with TTG (-33) and TAnnTTTG (-7) (Chen et al., 2007b).

TThese authors contributed equally to this work.

$\ddagger$ Present address: Center for Adaptation Genetics and Drug Resistance, Department of Molecular Biology and Microbiology, Tufts University School of Medicine, 136 Harrison Ave, Boston, MA 02111, USA.

Abbreviations: BT4001, Bacteroides thetaiotaomicron 4001; CAT, chloramphenicol acetyltransferase; RLU, relative light units.

A supplementary table, listing primers used in this study, is available with the online version of this paper.
In this study, the structure and function of a Bacteroides thetaiotaomicron 4001 (BT4001) 16S rRNA promoter was compared with that of an E. coli $16 \mathrm{~S}$ rRNA promoter from the $r r n B$ operon. In $E$. coli, transcription starts from two promoters (P1 and $\mathrm{P} 2)$ that are separated by approximately $150 \mathrm{bp}$, each containing -10 and -35 sites. $\mathrm{P} 1$ is located $\sim 350$ bp upstream, while P2 is $\sim 200$ bp upstream, from the $5^{\prime}$ end of the mature 16S rRNA. Three antitermination sequences located downstream of P2 are thought to allow efficient transcription of long, highly structured untranslated rRNA. E. coli rRNA synthesis is regulated by the collective response of the dual promoters to growth rate (Gourse et al., 1996), amino acid starvation (Cashel et al., 1996; Gafny et al., 1994) and rRNA gene dose (Gourse et al., 1996). P1 is considered the stronger of the two promoters since it accounts for most of rRNA transcription at all but the slowest growth rates (Sarmientos \& Cashel, 1983). It has been suggested that $\mathrm{P} 2$ promoters are inhibited by transcription from P1 (Gafny et al., 1994). P2 promoters are low-level constitutive promoters, responsible for most of the rRNA expression at low growth rates when transcription from P1 is reduced (Murray et al., 2003; Sarmientos \& Cashel, 1983). P1 of E. coli contains sequences upstream of its -35 hexamer, including an UP element (Rao et al., 1994; Ross et al., 1993) and Fis-binding sites (Ninnemann et al., 1992) that are responsible for strong stimulation of promoter activity, but are not required for growth rate regulation. P2 also contains an UP element; however, it has a much smaller effect on promoter activity than that exerted by the P1 UP element (Ross et al., 1993). Both $\mathrm{P} 1$ and $\mathrm{P} 2$ contain a $\mathrm{G}+\mathrm{C}$ rich 


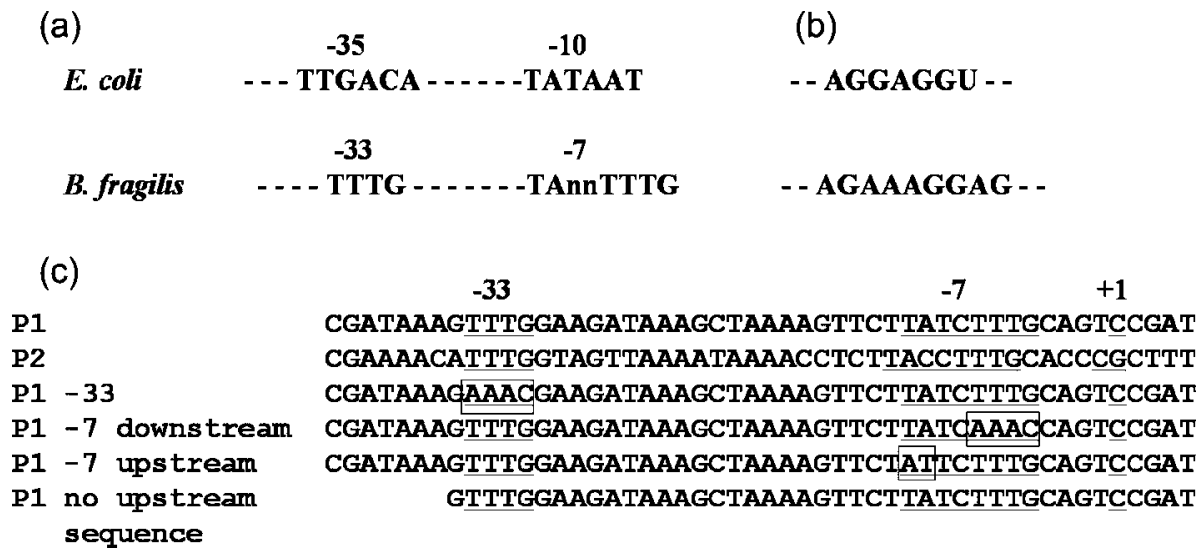

Fig. 1. Comparison of $E$. coli and B. fragilis promoter consensus sequences (a), Shine-Dalgarno sequences (b) and promoter substitutions (c). (a) Promoter consensus sequences of E. coli (Hawley \& McClure, 1983) and B. fragilis (Bayley et al., 2000). (b) Shine-Dalgarno sequences of $E$. coli (Weaver, 1999) and B. fragilis (Tribble et al., 1999). (c) Sequences of the -33 and -7 regions of the $\mathrm{P} 1$ and $\mathrm{P} 2$ promoters from $\mathrm{BT} 4001$ as well as the substitutions made in the P1 region of the 16S rRNA promoter. The -33 and -7 regions and the transcription initiation sites $(+1)$ of the $\mathrm{P} 1$ and $\mathrm{P} 2$ promoters are underlined. The spacing between the -33 and -7 regions is 21 and 20 bases for $P 1$ and $P 2$, respectively. Substitutions made in these two regions are boxed. The P1 promoter with no upstream sequence starts $1 \mathrm{bp}$ upstream of the first base of the -33 region.

region (Travers, 1984) between the -10 and the transcription start site that is required for stringent control (Josaitis et al., 1995; Murray et al., 2003).

A previous in vitro study examined the differences in transcription between $E$. coli and Bacteroides. It was observed that (1) Bacteroides fragilis $\sigma^{\mathrm{A}}$ factor could bind E. coli core RNA polymerase but did not allow transcription initiation, (2) purified Bacteroides core RNA polymerase plus $\sigma^{\mathrm{A}}$ was able to bind Bacteroides promoters but not E. coli promoters, and (3) Bacteroides core RNA polymerase with $E$. coli $\sigma^{70}$ could form a complex and promote expression of both E. coli and Bacteroides promoters, but with less efficiency than the native holoenzymes (Vingadassalom et al., 2005). Another in vitro study evaluated the structure of $\sigma^{\mathrm{A}}$ and its interactions with DNA (Vingadassalom et al., 2007).

Besides differences in promoter regions and sigma factor specificity, there is also a difference in the RBS between $E$. coli and Bacteroides. A canonical E. coli RBS is AGGAGGU (Fig. 1b; Weaver, 1999). Although the ribosomal machinery does not demand a precise distance, the optimal spacing between the Shine-Dalgarno sequence and the initiation codon is $8 \mathrm{nt}$ (Ringquist et al., 1992). A potential $B$. fragilis RBS, deduced from the $16 \mathrm{~S} \mathrm{rRNA}$, was published by Tribble et al. (1999) (Fig. 1b). Based on that sequence, potential RBSs were found within 3-9 nt upstream of the translation start site of a number of $B$. fragilis genes.

Our in vivo study complements the in vitro work done by others (Vingadassalom et al., 2005, 2007) and further demonstrates that gene expression capabilities between Bacteroides and E. coli depend on unique promoter structure. In our study the role of the RBSs of E. coli and Bacteroides has also been analysed. We developed two luminescence-based reporter systems that utilize the lux genes from the symbiotic luminescent soil bacterium Photorhabdus luminescens for use in Bacteroides species to comparatively examine the role of promoter elements and the RBS in controlling gene expression. These reporters will be useful tools for the Bacteroides-Cytophaga-Flavobacterium group.

\section{METHODS}

Bacterial strains, media and growth conditions. E. coli strains JM109 (Yanisch-Perron et al., 1985), DH5 $\alpha$ (Hanahan, 1983) and S17-1 (Simon et al., 1983) were grown aerobically in Luria-Bertani (LB) broth at $37^{\circ} \mathrm{C}$ with shaking (250 r.p.m.) and the appropriate antibiotics required for maintenance of the plasmids, $100 \mu \mathrm{g}$ ampicillin $\mathrm{ml}^{-1}$ (Ap100), $10 \mu \mathrm{g}$ tetracycline $\mathrm{ml}^{-1}$ (Tc10), $25 \mu \mathrm{g}$ chloramphenicol ml ${ }^{-1}(\mathrm{Cm} 25)$ or $100 \mu \mathrm{g} \quad$ kanamycin ml $\mathrm{m}^{-1}$ (Kan100). BT4001 (Shoemaker et al., 1996) was grown anaerobically at $37{ }^{\circ} \mathrm{C}$ in $10 \mathrm{ml}$ culture tubes containing trypticase yeast extract glucose broth (TYG; Holdeman \& Moore, 1975), $10 \mu \mathrm{g}$ erythromycin $\mathrm{ml}^{-1}(\mathrm{Em} 10)$ and $200 \mu \mathrm{g}$ gentamicin $\mathrm{ml}^{-1}(\mathrm{Gm} 200)$. Stock tubes of $B$. thetaiotaomicron were kept in $7 \mathrm{ml}$ PRAS chopped meat broth (Remel) for up to 2 weeks.

Isolation of a BT4001 16S rRNA promoter. As this work was initiated prior to publication of the genome sequence (Xu et al., 2003), an inverse PCR method was used to obtain the sequence of the region upstream of the 16S rRNA promoter. Primers $89 \mathrm{R}$ and $599 \mathrm{~F}$ (Supplementary Table S1) were then designed to amplify the putative BT4001 16S rRNA promoter from chromosomal DNA. The resulting PCR fragment was cloned into pGEM-T (Promega; pMLT2). Primers MLT2B and MLT2A (Supplementary Table S1) were used to add flanking PstI and BamHI sites to the end of the cloned PCR product (pMLT7).

Development of a bioluminescent gene reporter. Two reporters were constructed, pMDM and pMMT series vectors (Fig. 2), containing the entire $l u x$ operon (luxCDABE) or just containing 

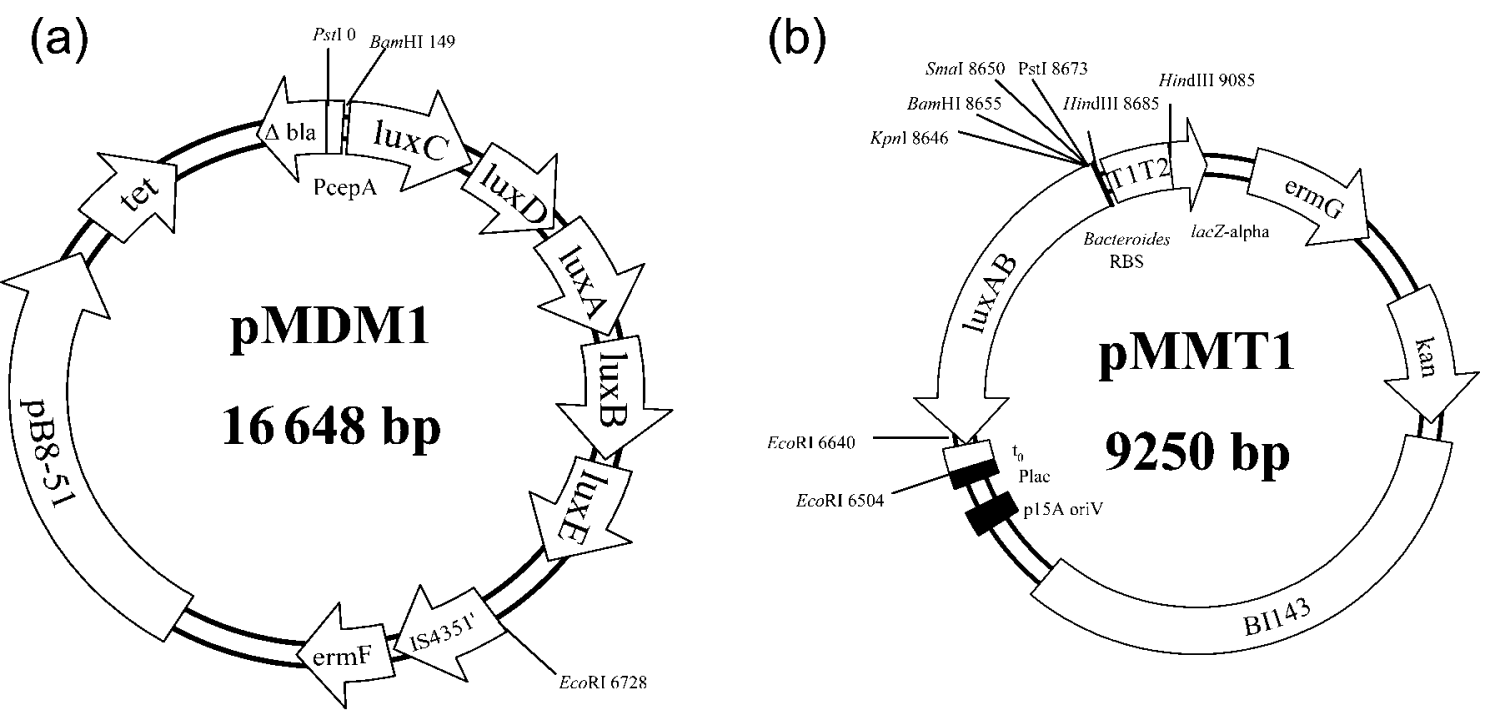

Fig. 2. Maps of two E. coli-Bacteroides shuttle/expression vectors. (a) pMDM1 is a fusion of pVAL1 with pJMP20 using the restriction sites EcoRI and Pstl. The cepA promoter was previously cloned into pJT205 via Pstl and BamHI, creating pJMP20. (b) pMMT1 contains luxAB with flanking $K p n l$ and EcoRl sites. The $\mathrm{t}_{0}$ transcription terminator and the $\mathrm{T}_{1} \mathrm{~T}_{2}$ transcription terminator are flanked by EcoRI and Hindlll restriction sites, respectively. The multiple cloning sequence contains four unique sites, Pstl, Smal, BamHI and Kpnl, which may be used for cloning additional promoters. See main text for details of construction.

$\operatorname{lu} x A B$, respectively. pMDM series vectors were constructed with the P. luminescens lux operon from pJT205 (Biotechnical Resources; Van Dyk \& Rosson, 1998). Promoters were first cloned directly into pJT205 using PstI and BamHI (New England Biolabs). The promoter and $l u x$ operon were ligated into the E. coli-Bacteroides shuttle vector pVAL1 (Valentine et al., 1988) using PstI and EcoRI sites.

A second series of vectors was created, for easy one-step cloning of promoters into the $l u x$ reporter. pMMT series $\operatorname{lux} A B$ constructs were made by cloning transcription terminators and the $\operatorname{lu} x A B$ cassette into pGWA48.3 (Whittle et al., 2002). The E. coli $r r n B \mathrm{~T}_{1} \mathrm{~T}_{2}$ transcription terminators were amplified from the plasmid pKK223-3 (Amann et al., 1983) using primers T1T2F3 and T1T2R3 (Supplementary Table S1) with flanking HindIII restriction sites. The $\mathrm{T}_{1} \mathrm{~T}_{2}$ transcription terminators were amplified via PCR, digested with HindIII, cloned into pUC19 (Yanisch-Perron et al., 1985) and subsequently cloned into pGWA48.3 (Whittle et al., 2002), creating pMDM7A-7. The lux $A B$ fragment was amplified from pJT205 (Van Dyk \& Rosson, 1998) using the primers LuxABFKpn or LuxABEcRBS (with the Bacteroides and E. coli RBSs, respectively) and LuxABREcoR2 (Supplementary Table S1), and cloned into pGEM-T. Both of these fragments were cloned into pMDM7A-7 using KpnI and EcoRI, creating pMLT15 (Bacteroides RBS) and pMLT16 (E. coli RBS). A second transcription terminator was used to prevent read-through transcription into the downstream region, where the origin of replication is located. The $\lambda$ phage $t_{0}$ transcription terminator was

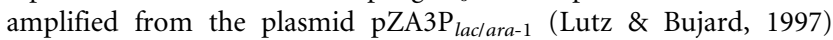
using primers T0F and T0R (Supplementary Table S1), which amplified the region with flanking EcoRI sites. It was cloned into pMLT15 and pMLT16, creating pMMT1 and pMMT2, with the Bacteroides and E. coli RBSs, respectively. The pMMT constructs contain four unique restriction sites (PstI, SmaI, BamHI and KpnI) for cloning promoters (Fig. 2b).

Cloning of 16S rRNA promoters and derivatives. BT4001 16S rRNA promoters $\mathrm{P} 1, \mathrm{P} 2$, and both $\mathrm{P} 1$ and $\mathrm{P} 2$ with no upstream sequence of $\mathrm{P} 1$, were isolated from pMLT7 via PCR, using primers listed in Supplementary Table S1, which introduced a PstI site upstream and a BamHI site downstream. Primers MLT2B and MLT7C were used for P1, MLT3A and MLT37B for P2, and MLT7A and MLT37B for P1 and P2 with no upstream sequences.

Substitutions were made to the P1 promoter region (Fig. 1c) using template pMLT12 and primers listed in Supplementary Table S1. The first round of PCR was done using DeepVent polymerase (New England Biolabs) with the forward primers 33P1, 7P1U and 7P1D, used for the $-33,-7$ upstream (TA) and -7 downstream (TTTG) substitutions, respectively, and the reverse primer MLT7C, which adds a BamHI site to the $3^{\prime}$ end. The $\mathrm{P} 1$ promoter with no upstream sequence was amplified using the primers MLT7A and MLT7C. The products were subsequently gel-extracted using a QIAquick Gel Purification kit (Qiagen) and used for a second round of PCR with the primer MLT2B, which adds a PstI site to the $5^{\prime}$ end, to obtain full-length P1.

Bacteroides promoters of interest were cloned into pMMT1 or pMMT2 via PstI and BamHI sites, while PstI and KpnI sites were used for the E. coli rrnB P1. See Table 1 for descriptions and designations of these promoter-reporter fusions.

Conjugation procedure. All of the E. coli-Bacteroides lux reporter/ shuttle vectors were transferred into BT4001 by diparental mating via E. coli strain S17-1 (Simon et al., 1983) using standard filter-mating protocols (Shoemaker et al., 1986).

In vitro luciferase assays. E. coli JM109 and BT4001 strains containing $l u x$ fusion constructs were grown to $\mathrm{OD}_{600} 0.5,1 \mathrm{ml}$ aliquots of each were sedimented by centrifugation and the pellets were stored at $-70{ }^{\circ} \mathrm{C}$. Luciferase assays using the harvested crude cell extracts were performed as previously described (Finney et al., 2002). In brief, each cell pellet was resuspended in $1 \mathrm{ml}$ lysis buffer (10 mM EDTA, pH 7.0, $10 \mathrm{mM}$ potassium phosphate, $5 \mathrm{mg}$ lysozyme, $1 \mathrm{mM}$ DTT, $0.1 \mathrm{~g}$ BSA per $100 \mathrm{ml}$ ). The samples were incubated at $-70{ }^{\circ} \mathrm{C}$ for $1 \mathrm{~h}$ and thawed at room temperature prior to analysis of the activity of aliquots. Activities were expressed as 
Table 1. Plasmids used in this study

\begin{tabular}{|c|c|c|}
\hline Plasmid & Relevance to study ${ }^{\star}$ & Source or reference \\
\hline pGEM-T & TA cloning vector used for sequencing, $A p^{R}$ & Promega \\
\hline pUC19 & Cloning vector used for $T_{1} T_{2}$ transcription terminator, $A p^{R}$ & Yanisch-Perron et al. (1985) \\
\hline pGWA48.3 & E. coli-Bacteroides shuttle vector, $\operatorname{Kan}^{\mathrm{R}}, \mathrm{Em}^{\mathrm{R}}$ & Whittle et al. (2002) \\
\hline pVAL1 & E. coli-Bacteroides shuttle vector, $\mathrm{Tc}^{\mathrm{R}}, \mathrm{Ap}^{\mathrm{R}}, \mathrm{Em}^{\mathrm{R}}$ & Valentine et al. (1988) \\
\hline pKK223-3 & Source of the $T_{1} T_{2}$ transcription terminator, $A p^{R}$ & Amann et al. (1983) \\
\hline pZA3P $P_{\text {laclara-1 }}$ & Source of the $\mathrm{t}_{0}$ transcription terminator, $\mathrm{Cm}^{\mathrm{R}}$ & Lutz \& Bujard (1997) \\
\hline pJT205 & Source of P. luminescens lux operon, Ap ${ }^{\mathrm{R}}$ & Van Dyk \& Rosson (1998) \\
\hline pFD711 & Source of wild-type $c e p A$ promoter, $\mathrm{Spc}^{\mathrm{R}}$ & Bayley et al. (2000) \\
\hline pFD714 & Source of variant cepA promoter ( $0 \%$ activity), $\mathrm{Spc}^{\mathrm{R}}$ & Bayley et al. (2000) \\
\hline pFD729 & Source of variant cepA promoter ( $33 \%$ activity), $\mathrm{Spc}^{\mathrm{R}}$ & Bayley et al. (2000) \\
\hline pMLT7 & Full-length BT4001 16S rRNA promoter, flanked by PstI and BamHI sites in pGEM-T & This study \\
\hline pMLT12 & BT4001 16S rRNA P1 flanked by PstI and BamHI sites in pGEM-T & This study \\
\hline pMLT15 & P. luminescens lux $A B$ with Bacteroides RBS downstream from $\mathrm{T}_{1} \mathrm{~T}_{2}$ in pMDM7A-7 & This study \\
\hline pMLT16 & P. luminescens lux $A B$ with $E$. coli $\mathrm{RBS}$ downstream from $\mathrm{T}_{1} \mathrm{~T}_{2}$ in pMDM7A-7 & This study \\
\hline pMDM1 & Wild-type cepA promoter construct, with entire $l u x$ operon in pVAL1†, $\mathrm{Tc}^{\mathrm{R}}, \mathrm{Ap}^{\mathrm{S}}, \mathrm{Em}^{\mathrm{R}}$ & This study \\
\hline pMDM4 & Promoterless lux operon reporter control for pMDM series $\dagger, \mathrm{Tc}^{\mathrm{R}}, \mathrm{Ap}^{\mathrm{S}}, \mathrm{Em}^{\mathrm{R}}$ & This study \\
\hline pMDM7A-7 & $\mathrm{T}_{1} \mathrm{~T}_{2}$ E. coli transcription terminators in pGWA48.3 & This study \\
\hline pMDM8 & BT4001 16S rRNA promoter-lux operon reporter construct in pVAL1 $\dagger, \mathrm{Tc}^{\mathrm{R}}, \mathrm{Ap}^{\mathrm{S}}, \mathrm{Em}^{\mathrm{R}}$ & This study \\
\hline pMMT1 & luxAB reporter system with the Bacteroides consensus $\mathrm{RBS}, \mathrm{Kan}^{\mathrm{R}}, \mathrm{Em}^{\mathrm{R}}$ & This study \\
\hline pMMT1A & E. coli rrnB $\mathrm{P} 1$ promoter in pMMT1 & This study \\
\hline pMMT1B & BT4001 full-length $16 \mathrm{~S}$ rRNA promoter in pMMT1 & This study \\
\hline pMMT1C & BT4001 16S rRNA P1 promoter in pMMT1 & This study \\
\hline pMMT1D & BT4001 16S rRNA P2 promoter in pMMT1 & This study \\
\hline pMMT1E & BT4001 P1P2 16S rRNA promoter with no upstream sequence in pMMT1 & This study \\
\hline pMMT1F & Wild-type cepA promoter in pMMT1 & This study \\
\hline pMMT1G & Variant cepA promoter construct ( $0 \%$ activity) in pMMT1 & This study \\
\hline pMMT1H & Variant cepA promoter construct ( $33 \%$ activity) in pMMT1 & This study \\
\hline pMMT1I & BT4001 16S rRNA P1 promoter with substituted -7 downstream region in pMMT1 & This study \\
\hline pMMT1J & BT4001 16S rRNA P1 promoter with a substituted -7 upstream region in pMMT1 & This study \\
\hline pMMT1K & BT4001 16S rRNA P1 promoter with a substituted -33 region in pMMT1 & This study \\
\hline pMMT1L & BT4001 16S rRNA P1 promoter with no upstream sequence in pMMT1 & This study \\
\hline pMMT2 & luxAB reporter system with the E. coli consensus RBS, $\operatorname{Kan}^{\mathrm{R}}, \mathrm{Em}^{\mathrm{R}}$ & This study \\
\hline pMMT2A & E. coli rrnB $\mathrm{P} 1$ promoter in $\mathrm{pMMT} 2$ & This study \\
\hline pMMT2B & BT4001 full-length $16 \mathrm{~S}$ rRNA promoter in pMMT2 & This study \\
\hline pMMT2C & BT4001 16S rRNA P1 promoter in pMMT2 & This study \\
\hline
\end{tabular}

${ }^{\star}$ For use in E. coli: $\mathrm{Tc}^{\mathrm{R}}$, tetracycline resistance; $\mathrm{Ap}^{\mathrm{R}}$, ampicillin resistance; $\mathrm{Tp}^{\mathrm{R}}$, trimethoprim resistance; $\mathrm{Kan}^{\mathrm{R}}$, kanamycin resistance; $\mathrm{Cm}^{\mathrm{R}}$, chloramphenicol resistance; $S p c^{\mathrm{R}}$, spectinomycin resistance; $\operatorname{Str}^{\mathrm{R}}$, streptomycin resistance. For use in Bacteroides: Rif ${ }^{\mathrm{R}}$, rifampicin resistance; $\mathrm{Gm}^{\mathrm{R}}$, gentamicin resistance; $\mathrm{Em}^{\mathrm{R}}$, erythromycin resistance.

$\dagger$ The ampicillin resistance gene is no longer functional in the pMDM series lux reporter.

relative light units (RLU) using a TD-20/20 luminometer (Turner Designs). The fold increase was defined as the RLU of the promoter construct divided by the RLU of the promoterless negative control.

In vivo whole-cell luminescence assays. Cellular luminescence was measured from BT4001 strains carrying the pMDM series as well as the pMMT1 constructs with the cepA promoter and two variant forms (Table 1). Assays were then performed as described by Phillips-Jones (1993). In brief, the cells were grown overnight in $10 \mathrm{ml} \mathrm{TYG} \mathrm{with} \mathrm{the}$ appropriate antibiotics, subcultured into fresh medium and grown to $\mathrm{OD}_{600}$ 0.5. A $1 \mu \mathrm{l}$ volume of $10 \%$ sonicated n-decyl aldehyde (decanal; Sigma) was added to $100 \mu \mathrm{l}$ live cells and the RLU were monitored over a period of $10 \mathrm{~min}$ using a TD-20/20 luminometer.

Primer extension. Primers BtPE1 and MLT37B (Supplementary Table S1) were used to examine the transcription start site of BT4001
P1 and P2, respectively. Each primer was $5^{\prime}$ end-labelled using $\left[\gamma-{ }^{32} \mathrm{P}\right] \mathrm{dATP}$ (Amersham) and a Primer Extension System kit (Promega). Primer extension was performed using this kit according to the manufacturer's instructions, except that an ethanol precipitation step was used to remove unincorporated $\left[\gamma_{-}{ }^{32} \mathrm{P}\right] \mathrm{dATP}$ from the primer. For ethanol precipitation, $90 \mu \mathrm{l}$ RNase-free water, $11 \mu \mathrm{l} 3 \mathrm{M}$ RNase-free sodium acetate and $220 \mu \mathrm{l}$ ethanol were added, mixed by vortexing, and kept at room temperature for $1 \mathrm{~h}$. Sequencing reactions of purified plasmids were performed using an fmol DNA cycle sequencing system kit (Promega) according to the manufacturer's instructions. Samples were analysed by electrophoresis in a $6 \%$ polyacrylamide-urea sequencing gel.

Statistics. Results were analysed using Instat 3 software (Graphpad Software) with an unpaired Student's $t$ test. A two-tailed $P$ value $<0.05$ was considered statistically significant. 
Efforts to improve the whole-cell assays. Efforts were made to develop high-throughput whole-cell luminescence assays. First, cells were grown on TYG plates followed by saturation of the plate with $10 \%$ sonicated decanal. Luminescence output was observed visually and by exposure of X-ray film. Second, isolated colonies were scraped off TYG plates and resuspended in a luminometer cuvette in $50 \mu \mathrm{l}$ luciferase assay buffer $(10 \mathrm{mM} \mathrm{KPO}, 1 \mathrm{mM}$ DTT, $0.1 \mathrm{~g}$ BSA per $100 \mathrm{ml}$ ). A $1 \mu 1$ volume of $10 \%$ sonicated decanal was added to the cell suspension and light output was monitored in a luminometer with an integration time of $4 \mathrm{~s}$. Finally, 96-well plate assays were performed with $200 \mu \mathrm{l}$ TYG agar plugs or broth cultures. Two or five microlitres of $10 \%$ sonicated decanal were added to broth and agar cultures, respectively. Light output was evaluated using a SpectraFluor Plus plate reader (Tecan), with a gain of either 100 or 125 and an integration time of $500 \mathrm{~ms}$.

\section{RESULTS}

\section{Development of the lux gene reporter system}

Preliminary work was done to evaluate the feasibility of using a lux-based reporter system in Bacteroides. The pMDM series reporters (Fig. 2a) were developed with the entire lux operon, encoding both the luciferase and the fatty acid reductase complex, and were used to measure the production of light using both whole-cell luminescence assays and crude cell extract luciferase assays. Expression of the reporter was examined using the cepA promoter (data not shown) or the BT4001 16S rRNA promoter (Fig. 3). BT4001 was able to produce a functional luciferase enzyme and produce light in the presence of oxygen using a lux operon reporter system under the control of either of the two Bacteroides promoters tested, but only when exogenous decanal substrate was added. The output of the negative control pMDM4 was negligible with respect to the background production of light in these two assay systems
(Fig. 3). However, there are concerns when using the pMDM series vectors. First, promoters are not easily cloned, because each promoter has to be cloned into pJT205 before the constructs can be fused with pVAL1. Second, the vectors are large, $\sim 17 \mathrm{~kb}$ in size. Third, strains carrying these large vectors have a roughly two- to threefold increase in generation time. Since the decanal substrate had to be supplied exogenously to the cells, the luxCDE genes were not necessary for the reporters. Therefore, the pMMT series vector system was developed as an improved reporter for Bacteroides because a shorter one-step cloning method could be used to introduce promoters into the vector using the unique BamHI, KpnI, PstI and SmaI sites. Besides easier manipulation of the vector, it is also smaller ( $\sim 10 \mathrm{~kb}$ in size) and has less of an effect on the growth of strains. In order to evaluate the sensitivity of the new pMMT lux reporter (Fig. 2b), prior to experiments with the as yet uncharacterized rRNA promoter, expression levels were measured from fusions to the well-characterized wild-type cepA promoter and two variants, which have decreased activity (0 and $33 \%$ activity), as demonstrated by the chloramphenicol acetyltransferase (CAT) gene reporter system (Bayley et al., 2000; Table 1). The strains carrying pMMT1F (wild-type), pMMT1G (0\%) and pMMT1H (33\%) were used for both whole-cell luminescence and crude cell extract luciferase assays (data not shown), which yielded similar results to the published CAT assays (Bayley et al., 2000). Hence, the new reporter was deemed to be sensitive enough to be used to analyse expression from $16 \mathrm{~S}$ rRNA constructs.

\section{Heterologous promoter expression}

Luciferase assays performed on cell extracts of E. coli demonstrated the activity of the E. coli $r r n B$ P1 promoter, (a)

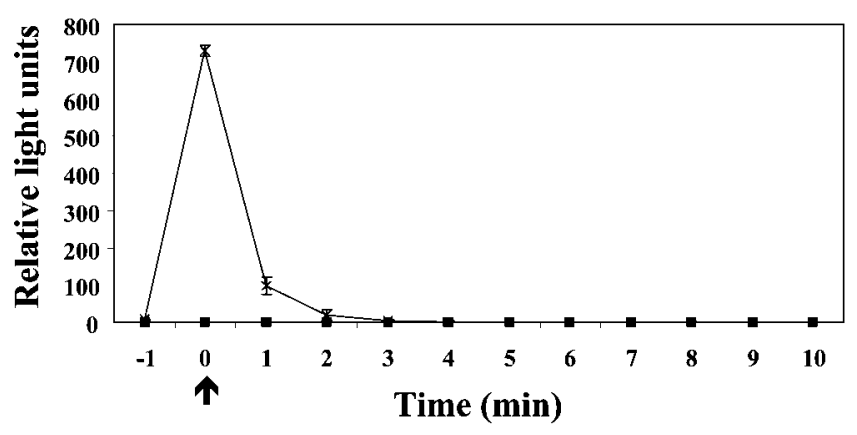

(b)

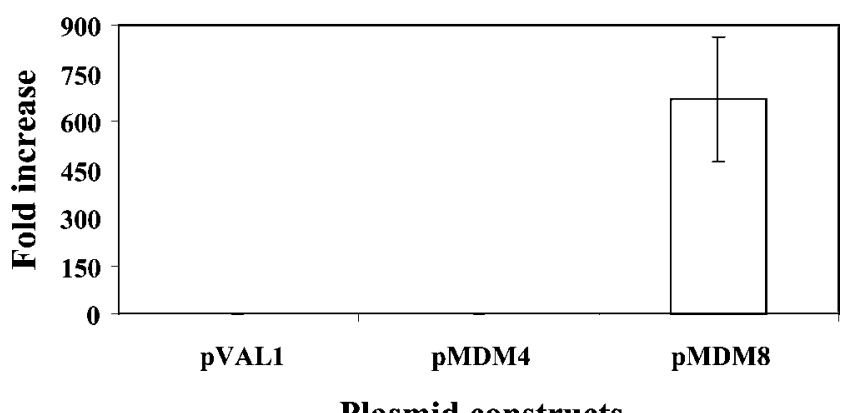

Plasmid constructs

Fig. 3. Luminescence and luciferase assays of BT4001 using the pMDM series $16 \mathrm{~S}$ rRNA promoter construct. (a) Qualitative studies of whole-cell luminescence assays performed on BT4001 strains containing the pMDM series constructs (refer to Table

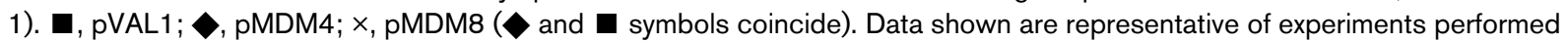
in two independent trials done in replicates of two. The black arrow at time 0 indicates the addition of decanal. (b) In vitro luciferase assays performed on crude cell extracts from strains containing the indicated vectors. Data are based on fold increase as compared with the expression levels of pMDM4. The mean value in RLU for pMDM4-containing cultures was $0.113 \pm 0.008$ from three independent trials performed in replicates of six. 
pMMT1A and pMMT2A, which expressed the lux reporter at 250-160-fold higher levels than the negative controls (Fig. 4a, b). rrnB P1 allowed expression of the lux reporter in the presence of either the Bacteroides or the E. coli RBS, but expression was slightly enhanced in the presence of the Bacteroides RBS. Differences in the spacing between the RBS and start codon being recognized in E. coli might explain the enhanced levels of expression observed from the pMMT1A construct (seven bases) when compared with pMMT2A (eight bases) (Fig. 4a, b). Luciferase assays on crude cell extract from E. coli also showed that, despite the presence of the E. coli RBS, the BT4001 full-length 16S rRNA promoter (pMMT2B) and 16S rRNA P1 (pMMT2C) were able to permit expression of the lux reporter at levels significantly higher than the negative control, but about an order of magnitude lower than the E. coli P1 promoter fusions (Fig. 4b). This demonstrates that differences in gene expression between E. coli and Bacteroides species occur at the level of promoter recognition.

Luciferase assays performed on cell extracts of BT4001 demonstrated the strength of the BT4001 16S rRNA promoter in that in the presence of its own RBS (pMMT1B), it permitted expression of the lux reporter at 260-fold higher levels than the promoterless control pMMT1 (Fig. 4c). Likewise, B. thetaiotaomicron $16 \mathrm{~S}$ rRNA P1 (pMMT1C) permitted expression of the reporter at 230fold higher levels than the negative control (Fig. 4c). E. coli $r r n B$ P1 was unable to drive expression of the lux reporter in BT4001, even in the presence of the Bacteroides RBS

\section{$\underline{\text { Ribosomal binding site }}$}
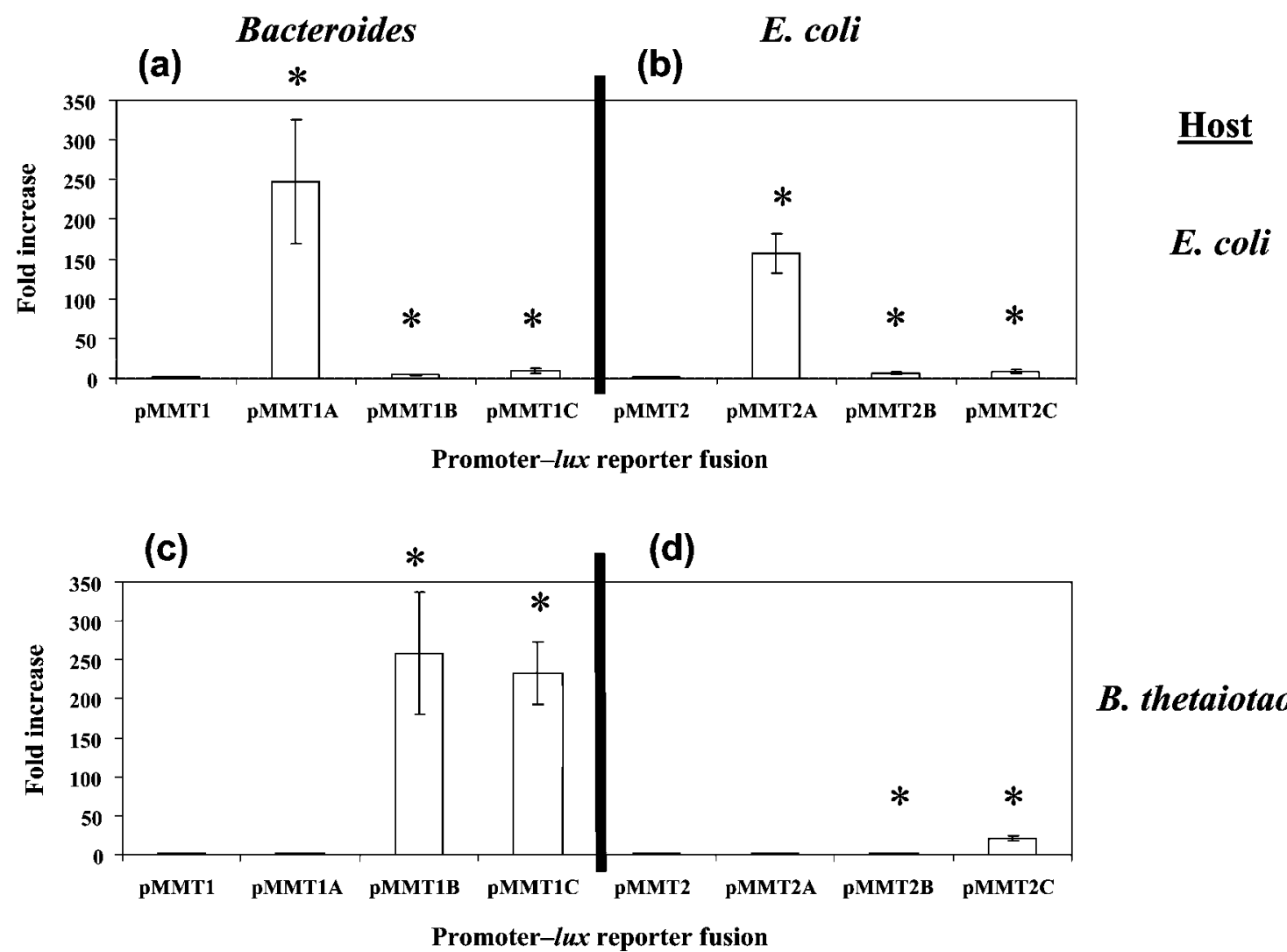

\section{B. thetaiotaomicron}

Fig. 4. Luciferase assays using both $E$. coli and $B$. thetaiotaomicron whole-cell lysates. $(a, b)$ Transcription levels from $E$. coli and BT4001 16S rRNA promoters using $E$. coli whole-cell lysates with the Bacteroides RBS (a) and the E. coli RBS (b). (c, d) Transcription initiation from E. coli and BT4001 16S rRNA promoters using BT4001 whole-cell lysates with the Bacteroides RBS (c) and the E. coli RBS (d). From left to right, the graphs show: pMMT1 or pMMT2, promoterless control with the Bacteroides RBS or E. coli RBS, respectively; pMMT1A or pMMT2A, E. coli rrnB P1; pMMT1B or pMMT2B, BT4001 fulllength 16S rRNA promoter; pMMT1C or pMMT2C, BT4001 16S rRNA P1. Results shown are the mean ( \pm SD) of two independent trials, performed in triplicate. The mean RLU values for the promoterless controls, for (a) and (b), were 0.71 and 0.13 , respectively. The mean RLU values for the promoterless controls, for (c) and (d), were 0.062 and 0.081 , respectively. Asterisks show statistically significant differences as compared with the promoterless controls, determined using Student's unpaired $t$ test (two-tailed); $P<0.001$. 
(pMMT1A; Fig. 4c). The Bacteroides promoters in combination with the E. coli RBS did permit initiation of luciferase expression at levels significantly higher than those for the negative control. In the presence of the E. coli RBS, BT4001 16S rRNA P1 allowed expression of the lux reporter at 20-fold higher levels than the promoterless control (pMMT2C; Fig. 4d). However, when compared with the strength of the BT4001 16S rRNA P1 promoter in the presence of the Bacteroides RBS (pMMT1C; Fig. 4c), this level of increase was about an order of magnitude lower. These results suggest that in Bacteroides species, translation initiation also plays a role in the disparity of gene expression between the two species.

\section{Analysis of a B. thetaiotaomicron 16S rRNA promoter region}

The lux reporter also allowed us to analyse the different regions of the BT4001 16S rRNA promoter to evaluate the role they play in initiating transcription. The BT4001 fulllength 16S rRNA promoter, P1, P2 and full-length promoter with no upstream sequences of P1 (pMMT1B, pMMT1C, pMMT1D and pMMT1E, respectively) were also examined (Fig. 5). In vitro luciferase assays demonstrated that the regions believed to contain BT4001 $16 \mathrm{~S}$ rRNA P1 and P2 contained a functional promoter, and that each was capable of permitting initiation of gene expression in the absence of the other (pMMT1C and pMMT1D, respectively; Fig. 5). This suggests that, as in E. coli, the two promoters work together in order to maintain the appropriate amount of rRNA in a cell at a given growth condition. Also, BT4001 full-length 16S rRNA promoter without any upstream sequences of P1 was capable of being expressed at levels close to that of the same promoter with upstream sequences (pMMT1E versus pMMT1B, respectively; Fig. 5).

Primer extension was done in order to examine the start site of transcription in Bacteroides. The results of these experiments showed that the transcription start site from the $\mathrm{P} 1$ promoter is a cytosine (Fig. $6 \mathrm{~b}$ ). The dominant start site of transcription for the $\mathrm{P} 2$ promoter is guanine with a secondary adjacent start site at a cytosine (Fig. 6a). These results indicate that the spacing between the centre of the -7 region and the transcription start site is $8 \mathrm{bp}$.

\section{Examination of the putative P1 $-33,-7$ and upstream sequences}

Substitutions were made in the Bacteroides $\mathrm{P} 1$ promoter to examine their effect on promoter strength. In vitro luciferase assays of crude cell extracts from cells that contained P1 (pMMT1C) detected 550-fold higher RLU than the promoterless control (pMMT1; data not shown). The activity levels of the other promoters as compared with the wild-type were: $11.2 \%$ for the -33 substituted (TTTG $\rightarrow$ AAAC), $4.0 \%$ for P1 with no upstream sequence, $1.8 \%$ for the -7 upstream substituted region $(\mathrm{TA} \rightarrow \mathrm{AT})$, and $0.44 \%$ for the -7 downstream substituted region $(\mathrm{TTTG} \rightarrow$ AAAC $) \quad($ pMMT1K, pMMT1L, pMMT1J and pMMT1I, respectively; data not shown). These results indicate that the -7 upstream and downstream sequences are equally important to the function of this promoter, more so than the -33 region, and that the region upstream of P1 influences promoter strength.

\section{Efforts to improve whole-cell luminescence assays}

Colonies containing pMDM1 on TYG plates flooded with decanal and exposed to X-ray film showed a very faint light emittance that could be seen by the naked eye, but lasted for less than a minute. Unfortunately, images on X-ray film were too diffuse to visualize individual colonies. When examining colonies resuspended in assay buffer, some light output above background was observed for up to $30 \mathrm{~min}$ from cells containing pMDM1 and for up to $60 \mathrm{~min}$ for cells containing pMMT1F and pMMT1H. These time limitations were presumably due to the decreased energy/

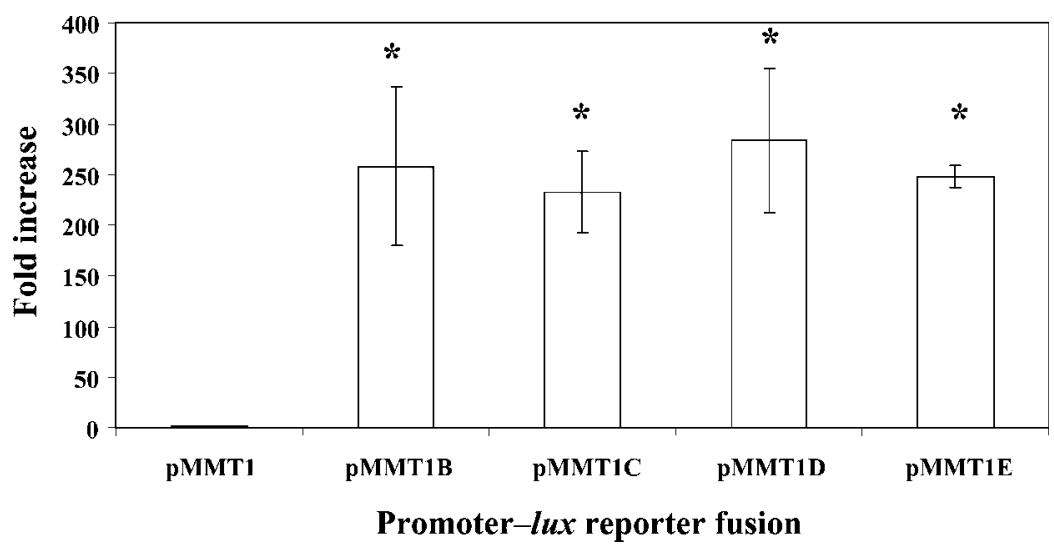

Fig. 5. Luciferase assays demonstrating transcription initiation from the different regions of the $B$. thetaiotaomicron 16S rRNA promoter. Results shown are the mean $( \pm S D)$ of two independent trials, performed in triplicate. From left to right, the graph shows: pMMT1, promoterless control; pMMT1B, BT4001 fulllength 16S rRNA promoter; pMMT1C, BT4001 16S rRNA P1; pMMT1D, BT4001 16S rRNA P2; pMMT1E, BT4001 16S rRNA $\mathrm{P} 1 \mathrm{P} 2$, with no upstream sequences. The RLU value for the promoterless control PMMT1 was 0.062 . Asterisks show statistically significant differences from the promoterless control, using Student's unpaired $t$ test (two-tailed); $P<0.001$. 
(a)

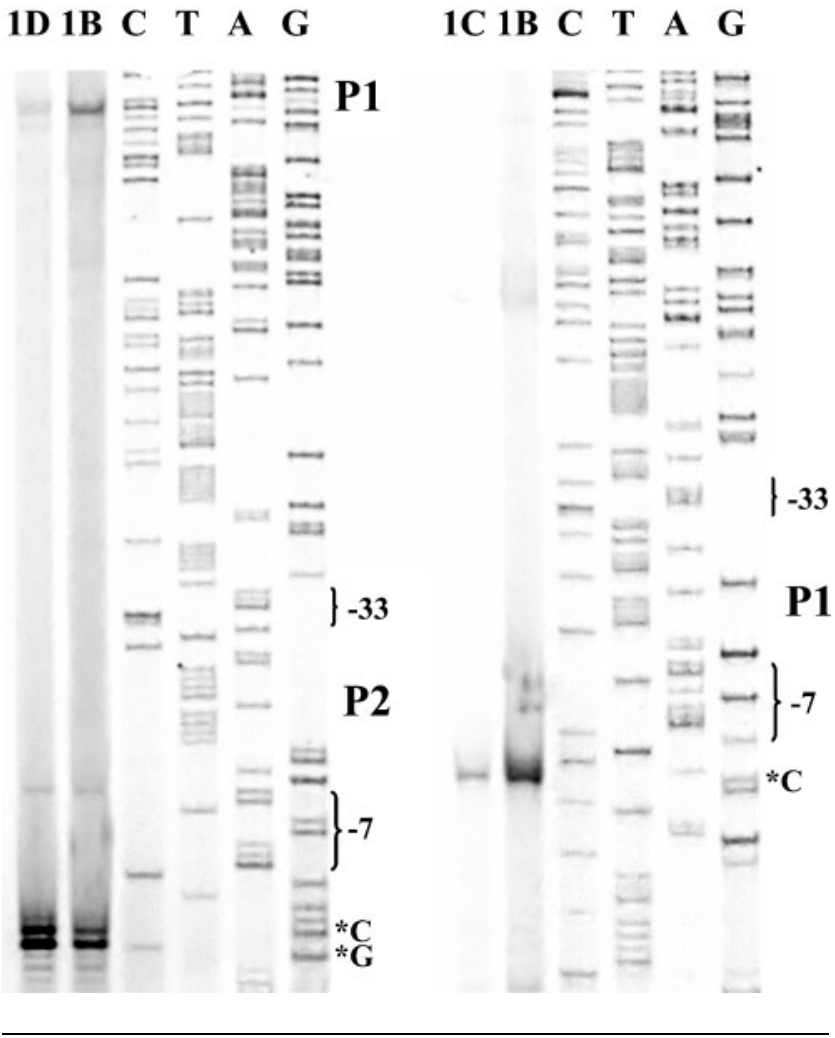

Fig. 6. Primer extension reactions mapping the transcription initiation sites of the $\mathrm{P} 1$ and $\mathrm{P} 2$ promoters. The lanes labelled $1 \mathrm{~B}, 1 \mathrm{C}$ and $1 \mathrm{D}$ correspond to primer extension products produced from transcripts derived from pMMT1B, pMMT1C and pMMT1D, respectively. (a) Expression from P2; (b) expression from P1. Sites of transcription initiation are marked by asterisks and the nucleotide at that position on the coding strand is indicated. The sequences for both $\mathrm{P} 1$ and $\mathrm{P} 2$ are shown in Fig. 1(c). The brightness/contrast of the lanes showing the results of the primer extension reactions and sequencing reactions were differentially enhanced using Adobe Photoshop.

reduced coenzyme levels of the cells upon exposure to oxygen.

The amount of light output from the broth cultures grown in 96-well plates containing strains with pMMT1F and pMMT1H was 2-15 times above the background levels for $\sim 30 \mathrm{~min}$. The background levels from the negative control cultures were 2- 5 RLU. However, with broth cultures containing pMDM1, the maximum light output was only four to six times above background for $5 \mathrm{~min}$ only, and the light output from these strains was very weak. When the cultures containing pMMT1F and pMMT1H were grown in the agar plugs, the RLU from these strains were above background for only about $10 \mathrm{~min}$. Therefore, highthroughput qualitative measurements to score for the presence of light output above background are possible for a short amount of time following the addition of the substrate. The best results screening for light output were achieved using the pMMT series vectors grown anaerobically in broth cultures on microtitre plates. However, downstream in vitro luciferase assays are necessary to accurately quantify promoter strength.

\section{DISCUSSION}

The barriers that impede successful expression of foreign bacterial genes in Bacteroides are still not fully understood. To date, most of the studies in the area of gene expression of Bacteroides have focused on a limited number of metabolic reporter systems. The four reporter systems that have previously been used in Bacteroides make use of $\beta$ glucuronidase (GUS) (Feldhaus et al., 1991), xylosidase/ arabinosidase (Whitehead, 1997), CAT (Bayley et al., 2000; Smith et al., 1992) and catechol 2,3-dioxygenase (XylE) (Chatzidaki-Livanis et al., 2008; Krinos et al., 2001). Endogenous enzyme production can be an issue with the first two systems, the substrate for the CAT assay can be cost-prohibitive, and only the $\mathrm{XylE}$ system is amenable to high-throughput differential screening. Thus, there is a need for the development of additional reporters with low background levels and rapid screening capabilities.

The new approach that we have used here involves the luminescence or lux operon as a tool for evaluating levels of gene expression in Bacteroides. To our knowledge, there is only one other study that has focused on using bioluminescence as a reporter system for an anaerobic bacterium. This study was performed on another human pathogen, Clostridium perfringens, using the lux operon from the marine bacterium Vibrio fischeri (Phillips-Jones, 1993). The problem with using the $V$. fischeri lux operon for studies in Bacteroides is that the Lux proteins are not fully active at temperatures above $30{ }^{\circ} \mathrm{C}$, while Bacteroides has an optimal growth temperature of $37^{\circ} \mathrm{C}$. To overcome this problem, the Lux system from $P$. luminescens, which functions at up to $45{ }^{\circ} \mathrm{C}$ (Szittner \& Meighen, 1990), was used for this study. Light output may be measured quantitatively over several orders of magnitude, making it an ideal reporter, but only under aerobic conditions.

Although Bacteroides are anaerobes, they are aerotolerant, capable of surviving in an oxygenated environment for a period of time, but are incapable of maintaining the activities of key enzymes necessary for energy production via central metabolism (Pan \& Imlay, 2001). Therefore, whole-cell luminescence assays (Fig. 3a) were found to be feasible on a limited time frame, where sufficient energy/ reduced coenzymes were available to produce the substrates $\mathrm{FMNH}_{2}$ and decanal necessary for luciferase activity. This problem was eliminated by adding the substrates exogenously during in vitro luciferase assays performed on crude cell extracts as described above. These reporters are useful tools for examining promoter activity and gene expression in BT4001 and could be applied to other species of Bacteroides, Cytophaga and Flavobacterium. 
The Bacteroides $16 \mathrm{~S}$ rRNA promoter region examined here has the same overall rRNA operon promoter structure as in E. coli. There are two BT4001 16S rRNA promoters, both containing the -7 and -33 Bacteroides consensus sequences, $\sim 150$ bp apart. Overall, there are five rRNA operons in BT4001 (Xu et al., 2003), four of which are 94-98\% identical in sequence. In these four, both $\mathrm{P} 1$ and $\mathrm{P} 2$ promoters are $100 \%$ conserved. The fifth rRNA operon is $88 \%$ identical to this sequence and only contains putative $\mathrm{P} 2$.

The results from the luciferase assays on cell extracts showed that BT4001 16S rRNA P1 and P2 promoters were both capable of initiating gene expression in the absence of the other and that the full-length $16 \mathrm{~S}$ rRNA promoter was able to initiate gene expression in the absence of any sequences upstream of the P1 promoter (Fig. 4). Estrem et al. (1998) identified a consensus UP element sequence in $E$. coli by using in vitro selection for upstream sequences that promote rapid RNA polymerase binding to the E. coli $r r n B$ P1 promoter, followed by in vivo screening for high promoter activity. UP element effects on promoter activity differ, correlating generally with the degree of similarity to the consensus sequence (Ross et al., 1998). A+T-rich upstream sequences have also been identified in compilations of Bacillus subtilis (Helmann, 1995) and Clostridium promoters (Graves \& Rabinowitz, 1986). Even though there is no sequence upstream from BT4001 16S rRNA P1 and P2 similar to the E. coli consensus UP element sequence, there appear to be $\mathrm{A}+\mathrm{T}$-rich regions. It has been demonstrated that sequences out to -54 of certain regulated $B$. fragilis promoters are required for optimal activity (Bayley et al., 2000), but this may reflect a requirement for activator binding. The examination of the region upstream of the BT4001 P1 promoter indicates that it is directly involved in its activity (data not shown), but this region does not seem to affect the activity of the full-length $16 \mathrm{~S}$ rRNA promoter (Fig. 5). Similar results have been observed in another member of the phylum Bacteroidetes, Flavobacterium. Deletion of the sequences upstream of the -33 region between -50 and -42 of the Flavobacterium johnsoniae ompA promoter leads to a dramatic reduction in promoter activity (Chen et al., 2007a). BT4001 has an A-rich region, $7 \mathrm{bp}$ in length, which is $12 \mathrm{bp}$ upstream of the -33 region of both $\mathrm{P} 1$ and P2. F. johnsoniae, however, has a T-rich region in the same area upstream of the ompA promoter

The Fis protein can bind DNA in a non-specific manner as well as at specific sites, making determination of a clear consensus sequence difficult (Betermier et al., 1994). Fisbinding sites could be present upstream of the BT4001 rRNA operon, but due to a weak consensus, further studies will need to be done to determine if they are present. However, sequence analysis of the BT4001 genome sequence (Xu et al., 2003) reveals no obvious Fis homologues.

A comparison of the $\mathrm{P} 116 \mathrm{~S}$ rRNA promoter variants with variants of the $B$. fragilis cepA promoter in the study by Bayley et al. (2000) confirmed the role of the -7 and -33 consensus sequences. With respect to the -7 downstream region (TTTG), both the cepA (Bayley et al., 2000) and the P1 substitutions show a lack of expression when this region is changed (Fig. 6). The P1 promoter with no upstream sequence and -33 substituted sequence helps confirm that the -33 region and the bases upstream of it are both important to the overall expression of the promoter. However, it seems that the upstream sequence has a larger effect on the activity of the promoter than the -33 region. These results also agree with earlier studies using the cepA promoter (Bayley et al., 2000). One discrepancy was seen in comparing the results from the $\mathrm{P} 1-7$ upstream region (TA) with those for the cepA promoter. We observed that the substitution of these two bases resulted in $1.8 \%$ activity as compared with the wild-type, unlike the cepA promoter, where a $38 \%$ activity was observed (Bayley et al., 2000). Perhaps the -7 upstream region (TA) is critical to the activity of BT4001 P1, but not to other promoters examined thus far, since those examined by Bayley et al. (2000) do not all share the consensus -7 upstream region. Our findings are similar to those of studies of Flavobacterium, where deletion of the -33 region of the ompA promoter (Chen $e t$ al., 2007b) or changes in either of the -7 upstream bases (TA) almost completely abolish gene expression (Chen et al., 2007a). Indeed, most substitutions at bases in both the -33 and the -7 region of the Flavobacterium promoter lead to decreased GFP reporter expression in comparison with the wild-type promoter levels (Chen et al., 2007a).

Overall, the results of this study expand on the work of others (Bayley et al., 2000; Vingadassalom et al., 2005, 2007) to provide additional insights into the requirements for transcription initiation in Bacteroides. Characterization of the P1 and P2 promoters and P1 promoter variants confirms the importance of the -33 and -7 sites for promoter activity in vivo. In E. coli, promoter recognition is the limiting factor in expressing Bacteroides genes (Fig. 4a, b). However, in Bacteroides, it seems that the E. coli promoters and RBS are not efficiently utilized by the Bacteroides transcription and translation machineries (Fig. $4 \mathrm{c}, \mathrm{d})$. Furthermore, it appears that the RBS sequence and spacing recognition by the ribosome in Bacteroides are more selective than those in E. coli.

\section{ACKNOWLEDGEMENTS}

We thank Jennifer Meador-Parton for her technical assistance, Reinhardt Rosson (Bio-Technical Resources, Manitowoc, WI) for providing pJT205, and Richard Gourse, Timothy Larson, Abigail Salyers, Nadja Shoemaker and C. Jeff Smith for the materials that they provided for this study. This work was supported by NSF CAREER award MCB-9875479, the Virginia Tech Optical Sciences and Engineering Research (OSER) Center and Carilion Biomedical Institute.

\section{REFERENCES}

Amann, E., Brosius, J. \& Ptashne, M. (1983). Vectors bearing a hybrid trp-lac promoter useful for regulated expression of cloned genes in Escherichia coli. Gene 25, 167-178. 
Bayley, D. P., Rocha, E. R. \& Smith, C. J. (2000). Analysis of cepA and other Bacteroides fragilis genes reveals a unique promoter structure. FEMS Microbiol Lett 193, 149-154.

Betermier, M., Galas, D. J. \& Chandler, M. (1994). Interaction of Fis protein with DNA: bending and specificity of binding. Biochimie 76, 958-967.

Cashel, M., Gentry, D. R., Hernandez, V. H. \& Vinella, D. (1996). The stringent response. In Escherichia coli and Salmonella typhimurium: Cellular and Molecular Biology, pp. 1458-1496. Edited by F. C. Neidhardt, R. Curtis III, J. L. Ingraham, E. C. C. Lin, K. B. Low, B. Magasanik, W. S. Reznikoff, M. Riley, M. Schaechter \& H. E. Umbarger. Washington, DC: American Society for Microbiology.

Chatzidaki-Livanis, M., Coyne, M. J., Roche-Hakansson, H. \& Comstock, L. E. (2008). Expression of a uniquely regulated extracellular polysaccharide confers a large-capsule phenotype to Bacteroides fragilis. J Bacteriol 190, 1020-1026.

Chen, S., Bagdasarian, M., Kaufman, M. G., Bates, A. K. \& Walker, E. D. (2007a). Mutational analysis of the ompA promoter from Flavobacterium johnsoniae. J Bacteriol 189, 5108-5118.

Chen, S., Bagdasarian, M., Kaufman, M. G. \& Walker, E. D. (2007b). Characterization of strong promoters from an environmental Flavobacterium hibernum strain by using a green fluorescent protein-based reporter system. Appl Environ Microbiol 73, 1089-1100.

Estrem, S. T., Gaal, T., Ross, W. \& Gourse, R. L. (1998). Identification of an UP element consensus sequence for bacterial promoters. Proc Natl Acad Sci U S A 95, 9761-9766.

Feldhaus, M. J., Hwa, V., Cheng, Q. \& Salyers, A. A. (1991). Use of an Escherichia coli $\beta$-glucuronidase gene as a reporter gene for investigation of Bacteroides promoters. J Bacteriol 173, 4540-4543.

Finney, A. H., Blick, R. J., Murakami, K., Ishihama, A. \& Stevens, A. M. (2002). Role of the C-terminal domain of the alpha subunit of RNA polymerase in LuxR-dependent transcriptional activation of the lux operon during quorum sensing. J Bacteriol 184, 4520-4528.

Gafny, R., Cohen, S., Nachaliel, N. \& Glaser, G. (1994). Isolated P2 rRNA promoters of Escherichia coli are strong promoters that are subject to stringent control. J Mol Biol 243, 152-156.

Gourse, R. L., Gaal, T., Bartlett, M. S., Appleman, J. A. \& Ross, W. (1996). rRNA transcription and growth rate-dependent regulation of ribosome synthesis in Escherichia coli. Annu Rev Microbiol 50, 645677.

Graves, M. C. \& Rabinowitz, J. C. (1986). In vivo and in vitro transcription of the Clostridium pasteurianum ferredoxin gene. Evidence for "extended" promoter elements in Gram-positive organisms. J Biol Chem 261, 11409-11415.

Hanahan, D. (1983). Studies on transformation of Escherichia coli with plasmids. J Mol Biol 166, 557-580.

Hawley, D. K. \& McClure, W. R. (1983). Compilation and analysis of Escherichia coli promoter DNA sequences. Nucleic Acids Res 11, 22372255.

Helmann, J. D. (1995). Compilation and analysis of Bacillus subtilis $\sigma^{\mathrm{A}}$-dependent promoter sequences: evidence for extended contact between RNA polymerase and upstream promoter DNA. Nucleic Acids Res 23, 2351-2360.

Holdeman, L. V. \& Moore, W. E. C. (1975). Anaerobe Laboratory Manual, 4th edn. Blacksburg, VA: Virginia Polytechnic Institute and State University.

Josaitis, C. A., Gaal, T. \& Gourse, R. L. (1995). Stringent control and growth-rate-dependent control have nonidentical promoter sequence requirements. Proc Natl Acad Sci U S A 92, 1117-1121.

Krinos, C. M., Coyne, M. J., Weinacht, K. G., Tzianabos, A. O., Kasper, D. L. \& Comstock, L. E. (2001). Extensive surface diversity of a commensal microorganism by multiple DNA inversions. Nature 414, $555-558$.

Lane, D. J. (1991). 16S/23S rRNA sequencing, In Nucleic Acid Techniques in Bacterial Systematics, pp. 115-175. Edited by E. Stackebrandt and M. Goodfellow. Chichester, New York: Wiley.

Lutz, R. \& Bujard, H. (1997). Independent and tight regulation of transcriptional units in Escherichia coli via the LacR/O, the TetR/O and AraC/ $\mathrm{I}_{1}-\mathrm{I}_{2}$ regulatory elements. Nucleic Acids Res 25, 1203-1210.

Murray, H. D., Appleman, J. A. \& Gourse, R. L. (2003). Regulation of the Escherichia coli rrnB P2 promoter. J Bacteriol 185, 28-34.

Ninnemann, O., Koch, C. \& Kahmann, R. (1992). The E. coli fis promoter is subject to stringent control and autoregulation. EMBO J 11, 1075-1083.

Pan, N. \& Imlay, J. A. (2001). How does oxygen inhibit central metabolism in the obligate anaerobe Bacteroides thetaiotaomicron. Mol Microbiol 39, 1562-1571.

Phillips-Jones, M. K. (1993). Bioluminescence (lux) expression in the anaerobe Clostridium perfringens. FEMS Microbiol Lett 106, 265-270.

Rao, L., Ross, W., Appleman, J. A., Gaal, T., Leirmo, S., Schlax, P. J., Record, M. T., Jr \& Gourse, R. L. (1994). Factor independent activation of $r r n B$ P1. An "extended" promoter with an upstream element that dramatically increases promoter strength. J Mol Biol 235, 1421-1435.

Ringquist, S., Shinedling, S., Barrick, D., Green, L., Binkley, J., Stormo, G. D. \& Gold, L. (1992). Translation initiation in Escherichia coli: sequences within the ribosome-binding site. Mol Microbiol 6, 1219-1229.

Ross, W., Gosink, K. K., Salomon, J., Igarashi, K., Zou, C., Ishihama, A., Severinov, K. \& Gourse, R. L. (1993). A third recognition element in bacterial promoters: DNA binding by the alpha subunit of RNA polymerase. Science 262, 1407-1413.

Ross, W., Aiyar, S. E., Salomon, J. \& Gourse, R. L. (1998). Escherichia coli promoters with UP elements of different strengths: modular structure of bacterial promoters. J Bacteriol 180, 5375-5383.

Sarmientos, P. \& Cashel, M. (1983). Carbon starvation and growth rate-dependent regulation of the Escherichia coli ribosomal RNA promoters: differential control of dual promoters. Proc Natl Acad Sci U S A 80, 7010-7013.

Shoemaker, N. B., Getty, C., Gardner, J. F. \& Salyers, A. A. (1986). Tn4351 transposes in Bacteroides spp. and mediates the integration of plasmid R751 into the Bacteroides chromosome. J Bacteriol 165, 929936.

Shoemaker, N. B., Wang, G. R. \& Salyers, A. A. (1996). The Bacteroides mobilizable insertion element, NBU1, integrates into the $3^{\prime}$ end of a Leu-tRNA gene and has an integrase that is a member of the lambda integrase family. J Bacteriol 178, 3594-3600.

Simon, R., Priefer, U. \& Puhler, A. (1983). A broad host range mobilization system for in vivo genetic engineering: transposon mutagenesis in Gram negative bacteria. Biotechnology (N Y) 1, 784791.

Smith, C. J., Rogers, M. B. \& McKee, M. L. (1992). Heterologous gene expression in Bacteroides fragilis. Plasmid 27, 141-154.

Szittner, R. \& Meighen, E. (1990). Nucleotide sequence, expression, and properties of luciferase coded by $l u x$ genes from a terrestrial bacterium. J Biol Chem 265, 16581-16587.

Travers, A. A. (1984). Conserved features of coordinately regulated $E$. coli promoters. Nucleic Acids Res 12, 2605-2618.

Tribble, G. D., Parker, A. C. \& Smith, C. J. (1999). Genetic structure and transcriptional analysis of a mobilizable, antibiotic resistance transposon from Bacteroides. Plasmid 42, 1-12. 
Valentine, P. J., Shoemaker, N. B. \& Salyers, A. A. (1988). Mobilization of Bacteroides plasmids by Bacteroides conjugal elements. J Bacteriol 170, 1319-1324.

Van Dyk, T. K. \& Rosson, R. A. (1998). Photorhabdus luminescens luxCDABE promoter probe vectors. Methods Mol Biol 102, 85-95.

Vingadassalom, D., Kolb, A., Mayer, C., Rybkine, T., Collatz, E. \& Podglajen, I. (2005). An unusual primary sigma factor in the Bacteroidetes phylum. Mol Microbiol 56, 888-902.

Vingadassalom, D., Kolb, A., Mayer, C., Collatz, E. \& Podglajen, I. (2007). Probing the importance of selected phylum-specific amino acids in $\sigma^{\mathrm{A}}$ of Bacteroides fragilis, a primary $\sigma$ factor naturally devoid of an N-terminal acidic region 1.1. J Biol Chem 282, 3442-3449.

Weaver, R. (1999). The mechanism of translation I: initiation. In Molecular Biology, pp. 531-564. Boston, MA: McGraw-Hill.

Werner, H. (1974). Differentiation and medical importance of saccharolytic intestinal Bacteroides. Arzneimittelforschung 24, 340-343.
Whitehead, T. R. (1997). Development of a bifunctional xylosidase/ arabinosidase gene as a reporter gene for the Gram-negative anaerobes Bacteroides and Porphyromonas, and Escherichia coli. Curr Microbiol 35, 282-286.

Whittle, G., Shoemaker, N. B. \& Salyers, A. A. (2002). Characterization of genes involved in modulation of conjugal transfer of the Bacteroides conjugative transposon CTnDOT. J Bacteriol 184, 3839-3847.

Xu, J., Bjursell, M. K., Himrod, J., Deng, S., Carmichael, L. K., Chiang, H. C., Hooper, L. V. \& Gordon, J. I. (2003). A genomic view of the human-Bacteroides thetaiotaomicron symbiosis. Science 299, 20742076.

Yanisch-Perron, C., Vieira, J. \& Messing, J. (1985). Improved M13 phage cloning vectors and host strains: nucleotide sequences of the M13mp18 and pUC19 vectors. Gene 33, 103-119.

Edited by: H. J. Flint 